J. Indones. Math. Soc.

Vol. 19, No. 2 (2013), pp. 67-77.

\title{
SUPER EAT LABELINGS OF SUBDIVIDED STARS
}

\author{
M. JAVAID ${ }^{1}$ AND A.A. BhATTI ${ }^{2}$ \\ ${ }^{1,2}$ Department of Mathematics \\ National University of Computer and \\ Emerging Sciences Lahore, Pakistan. \\ javaidmath@gmail.com \\ akhlaq.ahmad@nu.edu.pk
}

\begin{abstract}
Kotzig and Rosa (1970) conjectured that every tree admits edge-magic total labeling. Enomoto et al. (1998) proposed the conjecture that every tree is super edge-magic total. In this paper, we describe super $(a, d)$-edge-antimagic total labelings on a subclass of the subdivided stars denoted by $T\left(n, n, n, n, n_{5}, n_{6} \ldots, n_{r}\right)$ for $d \in\{0,1,2\}$, where $n \geq 3$ odd, $r \geq 5$ and $n_{m}=2^{m-4}(n-1)+1$ for $5 \leq m \leq r$. Key words: Super $(a, d)$-EAT labelings, subdivision of stars.
\end{abstract}

\begin{abstract}
Abstrak. Kotzig dan Rosa (1970) telah membuat konjektur bahwa setiap tree dapat menghasilkan edge-magic total labeling. Enomoto et al. (1998) telah membuat konjektur bahwa setiap tree adalah super edge-magic total. Di dalam makalah ini, kami menjelaskan super $(a, d)$-edge-antimagic total labeling pada sebuah sub-kelas dari star yang terbagi yang dinyatakan oleh $T\left(n, n, n, n, n_{5}, n_{6} \ldots, n_{r}\right)$ untuk $d \in$ $\{0,1,2\}$, dimana $n \geq 3$ ganjil, $r \geq 5$ dan $n_{m}=2^{m-4}(n-1)+1$ untuk $5 \leq m \leq r$. Kata kunci: Super $(a, d)-E A T$ labelings, pembagian stars.
\end{abstract}

\section{INTRODUCTION}

All graphs in this paper are finite, undirected and simple. For a graph $G, V(G)$ and $E(G)$ denote the vertex-set and the edge-set, respectively. A $(v, e)$-graph $G$ is a graph such that $|V(G)|=v$ and $|E(G)|=e$. A general reference for graphtheoretic ideas can be seen in [29]. A labeling (or valuation) of a graph is a map that carries graph elements to numbers (usually to positive or non-negative integers). In this paper, the domain will be the set of all vertices and edges and such a labeling is called a total labeling. Some labelings use the vertex-set only or the

2000 Mathematics Subject Classification: 05C78 37M10.

Received: 17-11-2012, revised: 27-05-2013, accepted: 06-06-2013. 
edge-set only and we shall call them vertex-labelings or edge-labelings, respectively. A number of classification studies on edge antimagic total graphs has been intensively investigated. For further studies on antimagic labelings, reader can see $[13,5]$.

Definition 1.1 A $(s, d)$-edge-antimagic vertex $((s, d)$-EAV) labeling of a graph $G$ is a bijective function $\lambda: V(G) \rightarrow\{1,2, \ldots, v\}$ such that the set of edge-sums of all edges in $G,\{w(x y)=\lambda(x)+\lambda(y): x y \in E(G)\}$, forms an arithmetic progression $\{s, s+d, s+2 d, \ldots, s+(e-1) d\}$, where $s>0$ and $d \geq 0$ are two fixed integers.

Definition 1.2. An $(a, d)$-edge-antimagic total $((a, d)$-EAT) labeling of a graph $G$ is a bijective function $\lambda: V(G) \cup E(G) \rightarrow\{1,2, \ldots, v+e\}$ such that the set of edge-weights of all edges in $G,\{w(x y)=\lambda(x)+\lambda(x y)+\lambda(y): x y \in E(G)\}$, forms an arithmetic progression $\{a, a+d, a+2 d, \ldots, a+(e-1) d\}$, where $a>0$ and $d \geq 0$ are two fixed integers. If such a labeling exists then $G$ is said to be an $(a, d)$-EAT graph. Additionally, if $\lambda(V(G))=\{1,2, \ldots, v\}$ then $\lambda$ is called a super $(a, d)$-edge-antimagic total (super $(a, d)$-EAT) labeling and $G$ becomes a super $(a, d)$-EAT graph.

In the above definition, if $d=0$ then $(a, 0)$-EAT labeling is called edge-magic total (EMT) labeling and super $(a, 0)$-EAT labeling is called super edge-magic total (SEMT) labeling. The subject of edge-magic total (EMT) labeling of graphs has its origin in the works of Kotzig and Rosa $[20,21]$ on what they called magic valuations of graphs. The definition of $(a, d)$-EAT labeling was introduced by Simanjuntak, Bertault and Miller in [27] as a natural extension of EMT labeling defined by Kotzig and Rosa. A super $(a, d)$-EAT labeling is a natural extension of the notion of SEMT labeling defined by Enomoto, Lladó, Nakamigawa and Ringel in [9]. Moreover, they proposed the following conjecture:

Conjecture 1.1 Every tree admits SEMT labeling [9].

In the favour of this conjecture, many authors have proved the existence of SEMT labelings for various particular classes of trees for examples [1-8, 10-12, 14-17, 24, $25,28,29]$. Lee and Shah [22] verified this conjecture by a computer search for trees with at most 17 vertices. However, this conjecture is still open. Bača et al. investigated the following relationship between $(s, d)$-EAV labeling and $(a, d)$-EAT labeling [3]:

Proposition 1.1. If a $(v, e)$-graph $G$ has a $(s, d)$-EAV labeling then $G$ admits

(i) a super $(s+v+1, d+1)$-EAT labeling,

(ii) a super $(s+v+e, d-1)$-EAT labeling. 
The notion of dual labeling has been introduced by Wallis [30]. The next lemma follows from the principal of duality, which is first studied by Baskoro [8].

Lemma 1.1 If $g$ is a super edge-magic total labeling of $G$ with the magic constant $c$, then the function $g_{1}: V(G) \cup E(G) \rightarrow\{1,2, \ldots, v+e\}$ defined by

$$
g_{1}(x)= \begin{cases}v+1-g(x), & \text { for } x \in V(G), \\ 2 v+e+1-g(x), & \text { for } x \in E(G),\end{cases}
$$

is also a super-magic total labeling of $G$ with the magic constant $c_{1}=4 v+e+3-c$.

Definition 1.3 For $n_{i} \geq 1$ and $r \geq 2$, let $G \cong T\left(n_{1}, n_{2}, \ldots, n_{r}\right)$ be a graph obtained by inserting $n_{i}-1$ vertices to each of the $i^{t h}$ edge of the star $K_{1, r}$, where $1 \leq i \leq r$. Thus, the graph $T \underbrace{(1,1, \ldots, 1)}_{r-\text { times }}$ is a star $K_{1, r}$.

Subdivided stars form a particular class of trees and many authors have proved the antimagicness for various subclasses of subdivided stars as follows:

- $\mathrm{Lu}[23,24]$ has called the subdivided star $T(m, n, k)$ as a three-path tree. Moreover, he has proved that it is a SEMT graph if $n$ and $m$ are odd with $k=n+1$ or $k=n+2$.

- Ngurah et al. [25] have proved that $T(m, n, k)$ is a SEMT graph if $n$ and $m$ are odd with $k=n+3$ or $k=n+4$.

- In [26], Salman et al. have found the results related to SEMT labelings on the subdivision of stars $S_{n}^{m}$ for $m=1,2$, where $S_{n}^{1} \cong T \underbrace{(2,2, \ldots, 2)}_{n-\text { times }}$ and

$$
S_{n}^{2} \cong T \underbrace{(3,3, \ldots, 3)}_{n-\text { times }} .
$$

- In [16], Javaid et al. have formulated SEMT labelings on the subdivision of star $K_{1,4}$ and w-trees.

- Javaid and Akhlaq [17] have proved that the subdivided stars $T(n, n, n+$ $\left.2, n+2, n_{5}, \ldots, n_{p}\right)$ admit super $(a, d)$-EAT labelings, where $n \geq 3$ is odd, $r \geq 5$ and $n_{m}=1+(n+1) 2^{m-4}$ for $5 \leq m \leq r$.

However, the problem to find super $(a, d)$-EAT labelings on $T\left(n_{1}, n_{2}, n_{3}, \ldots, n_{r}\right)$ for different $\left\{n_{i}: 1 \leq i \leq r\right\}$ is still open. In this paper, for $d \in\{0,1,2\}$, we find super $(a, d)$-EAT labelings on the subdivided stars $T\left(n, n, n, n, n_{5}, n_{6}, \ldots, n_{r}\right)$, where $n \geq 3$ is odd, $r \geq 5$ and $n_{m}=2^{m-4}(n-1)+1$ for $5 \leq m \leq r$.

\section{BOUNDS OF MAGIC CONSTANT}

In this section, we present different lemmas related to lower and upper bounds of the magic constant $a$ for various subclasses of subdivided stars. 
Ngurah et al. [25] found the following lower and upper bounds of the magic constant $a$ for a particular subclass of the subdivided stars denoted by $T(m, n, k)$, which is given below:

Lemma 2.1. If $T(m, n, k)$ is a super $(a, 0)$-EAT graph, then $\frac{1}{2 l}\left(5 l^{2}+3 l+6\right) \leq$ $a \leq \frac{1}{2 l}\left(5 l^{2}+11 l-6\right)$, where $l=m+n+k$.

The lower and upper bounds of the magic constant $a$ for a particular subclass of the subdivided stats $T \underbrace{(n, n, \ldots, n)}_{n-t \text { times }}$ are established by Salman et al. [26] as follows:

Lemma 2.2. If $T \underbrace{(n, n, \ldots, n)}_{n-\text { times }}$ is a super $(a, 0)$-EAT graph, then $\frac{1}{2 l}\left(5 l^{2}+(9-\right.$ $\left.2 n) l+n^{2}-n\right) \leq a \leq \frac{1}{2 l}\left(5 l^{2}+(2 n+5) l+n-n^{2}\right)$, where $l=n^{2}$.

Javaid [19] has proved lower and upper bounds of the magic constant $a$ for the most extended subclasses of the subdivided stars denoted by $T\left(n_{1}, n_{2}, n_{3}, \ldots, n_{r}\right)$ with any $n_{i} \geq 1$ for $1 \leq i \leq r$, which is presented in the following lemma:

Lemma 2.3. If $T\left(n_{1}, n_{2}, n_{3}, \ldots, n_{r}\right)$ is a super $(a, 0)$-EAT graph, then $\frac{1}{2 l}\left(5 l^{2}+r^{2}-\right.$ $2 l r+9 l-r) \leq a \leq \frac{1}{2 l}\left(5 l^{2}-r^{2}+2 l r+5 l+r\right)$, where $l=\sum_{i=1}^{r} n_{i}$.

\section{SUPER $(a, d)$-EAT LABELINGS OF SUBDIVIDED STARS}

In this section, we prove the main results related to super $(a, d)$-EAT labelings on a particular subclass of the subdivided stars for different values of the parameter $d$.

Theorem 2.1. For any odd $n \geq 3, G \cong T(n, n, n, n, 2 n-1)$ admits a super $(a, 0)$ EAT labeling with $a=2 v+s-1$ and a super $(a, 2)$-EAT labeling with $a ́=v+s+1$, where $v=|V(G)|$ and $s=3 n+4$.

Proof. Let us denote the vertices and edges of $G$, as follows:

$V(G)=\{c\} \cup\left\{x_{i}^{l_{i}} \mid 1 \leq i \leq 5 ; 1 \leq l_{i} \leq n_{i}\right\}, E(G)=\left\{c x_{i}^{1} \mid 1 \leq i \leq 5\right\} \cup$ $\left\{x_{i}^{l_{i}} x_{i}^{l_{i}+1} \mid 1 \leq i \leq 5 ; 1 \leq l_{i} \leq n_{i}-1\right\}$. If $v=|V(G)|$ and $e=|E(G)|$ then $v=6 n$ and $e=6 n-1$. Now, we define the labeling $\lambda: V(G) \rightarrow\{1,2, \ldots, v\}$ as follows:

$$
\lambda(c)=4 n+2
$$


For

$1 \leq l_{i} \leq n_{i}$ odd

$$
\lambda(u)= \begin{cases}\frac{l_{1}+1}{2}, & \text { for } u=x_{1}^{l_{1}}, \\ (n+2)-\frac{l_{2}+1}{2}, & \text { for } u=x_{2}^{l_{2}}, \\ (n+1)+\frac{l_{3}+1}{2}, & \text { for } u=x_{3}^{l_{3}}, \\ (2 n+3)-\frac{l_{4}+1}{2}, & \text { for } u=x_{4}^{l_{4}} \\ (3 n+3)-\frac{l_{5}+1}{2}, & \text { for } u=x_{5}^{l_{5}} .\end{cases}
$$

For $\quad 2 \leq l_{i} \leq n_{i}-1$ even;

$$
\lambda(u)= \begin{cases}(3 n+2)+\frac{l_{1}}{2}, & \text { for } u=x_{1}^{l_{1}}, \\ (4 n+2)-\frac{l_{2}}{2}, & \text { for } u=x_{2}^{l_{2}}, \\ (4 n+2)+\frac{l_{3}}{2}, & \text { for } u=x_{3}^{l_{3}}, \\ (5 n+2)-\frac{l_{4}}{2}, & \text { for } u=x_{4}^{l_{4}}, \\ (6 n+1)-\frac{l_{5}}{2}, & \text { for } u=x_{5}^{l_{5}} .\end{cases}
$$

The set of all edge-sums generated by the above formulas forms a consecutive integer sequence $s=3 n+4,3 n+5, \cdots, 3 n+3+e$. Therefore, by Proposition 1.1, $\lambda$ can be extended to a super $(a, 0)$-EAT labeling with magic constant $a=2 v+s-1=15 n+3$ and to a super $(\dot{a}, 2)$-EAT labeling with minimum edge-weight $a ́=v+1+s=9 n+5$.

Theorem 2.2. For any odd $n \geq 3, G \cong T(n, n, n, n, 2 n-1)$ admits a super $(a, 1)$-EAT labeling with $a=s+\frac{3 v}{2}$, where $v=|V(G)|$ and $s=3 n+4$.

Proof. Let us consider the vertex and edge set of $G$ and the labeling $\lambda: V(G) \rightarrow$ $\{1,2, \ldots, v\}$ by the same manner as in Theorem 2.1. It follows that edge-sums of all the edges of $G$ constitute an arithmetic sequence $3 n+4,3 n+5, \cdots, 3 n+3+e$, with common difference 1 . We denote it by $A=\left\{a_{i} ; 1 \leq i \leq e\right\}$. Now to show that $\lambda$ is an $(a, 1)$-EAT labeling of $G$, define the set of edge-labels as $B=\left\{b_{j}=v+j ; 1 \leq\right.$ $j \leq e\}$. The set of edge-weights can be obtained as $C=\left\{a_{2 i-1}+b_{e-i+1} ; 1 \leq i \leq\right.$ $\left.\frac{e+1}{2}\right\} \cup\left\{a_{2 j}+b_{\frac{e-1}{2}-j+1} ; 1 \leq j \leq \frac{e+1}{2}-1\right\}$. It is easy to see that $C$ constitutes an arithmetic sequence with $d=1$ and $a=s+\frac{3 v}{2}=12 n+4$. Since all vertices receive the smallest labels, $\lambda$ is a super $(a, 1)$-EAT labeling.

Theorem 2.3. For any odd $n \geq 3, G \cong T(n, n, n, n, 2 n-1,4 n-3)$ admits a super $(a, 0)$-EAT labeling with $a=2 v+s-1$ and a super $(\dot{a}, 2)$-EAT labeling with $\dot{a}=v+s+1$, where $v=|V(G)|$ and $s=5 n+3$. 
Proof. Let us denote the vertices and edges of $G$, as follows:

$V(G)=\{c\} \cup\left\{x_{i}^{l_{i}} \mid 1 \leq i \leq 6 ; 1 \leq l_{i} \leq n_{i}\right\}, E(G)=\left\{c x_{i}^{1} \mid 1 \leq i \leq 6\right\} \cup$ $\left\{x_{i}^{l_{i}} x_{i}^{l_{i}+1} \mid 1 \leq i \leq 6 ; 1 \leq l_{i} \leq n_{i}-1\right\}$. If $v=|V(G)|$ and $e=|E(G)|$ then $v=$ $10 n-3$, and $e=10 n-4$. Now, we define the labeling $\lambda: V(G) \rightarrow\{1,2, \ldots, v\}$ as follows:

$$
\lambda(c)=6 n+1
$$

For $\quad 1 \leq l_{i} \leq n_{i}$ odd

$$
\lambda(u)= \begin{cases}\frac{l_{1}+1}{2}, & \text { for } u=x_{1}^{l_{1}}, \\ (n+2)-\frac{l_{2}+1}{2}, & \text { for } u=x_{2}^{l_{2}}, \\ (n+1)+\frac{l_{3}+1}{2}, & \text { for } u=x_{3}^{l_{3}}, \\ (2 n+3)-\frac{l_{4}+1}{2}, & \text { for } u=x_{4}^{l_{4}} \\ (3 n+3)-\frac{l_{5}+1}{2}, & \text { for } u=x_{5}^{l_{5}}, \\ (5 n+2)-\frac{l_{6}+1}{2}, & \text { for } u=x_{6}^{l_{6}} .\end{cases}
$$

For $\quad 2 \leq l_{i} \leq n_{i}-1$ even;

$$
\lambda(u)= \begin{cases}(5 n+1)+\frac{l_{1}}{2}, & \text { for } u=x_{1}^{l_{1},} \\ (6 n+1)-\frac{l_{2}}{2}, & \text { for } u=x_{2}^{l_{2},} \\ (6 n+1)+\frac{l_{3}}{2}, & \text { for } u=x_{3}^{l_{3},} \\ (7 n+1)-\frac{l_{4}}{2}, & \text { for } u=x_{4}^{l_{4},} \\ 8 n-\frac{l_{5}}{2}, & \text { for } u=x_{5}^{l_{5}}, \\ (10 n-2)-\frac{l_{6}}{2}, & \text { for } u=x_{6}^{l_{6} .} .\end{cases}
$$

The set of all edge-sums generated by the above formulas forms a consecutive integer sequence $s=5 n+3,5 n+4, \cdots, 5 n+2+e$. Therefore, by Proposition $1.1, \lambda$ can be extended to a super $(a, 0)$-EAT labeling with magic constant $a=2 v+s-1=25 n-4$ and to a super $(a, 2)$-EAT labeling with minimum edgeweight $a ́=v+1+s=15 n+1$.

Theorem 2.4. For any odd $n \geq 3, G \cong T(n, n, n, n, 2 n-1,4 n-3,8 n-7)$ admits a super $(a, 0)$-EAT labeling with $a=2 v+s-1$ and a super (á,2)-EAT labeling with $a=v+s+1$, where $v=|V(G)|$ and $s=9 n$.

Proof. Let us denote the vertices and edges of $G$, as follows:

$V(G)=\{c\} \cup\left\{x_{i}^{l_{i}} \mid 1 \leq i \leq 7 ; 1 \leq l_{i} \leq n_{i}\right\}, \quad E(G)=\left\{c x_{i}^{1} \mid 1 \leq i \leq 7\right\} \cup$ $\left\{x_{i}^{l_{i}} x_{i}^{l_{i}+1} \mid 1 \leq i \leq 7 ; 1 \leq l_{i} \leq n_{i}-1\right\}$. If $v=|V(G)|$ and $e=|E(G)|$ then $v=$ 
$18 n-10$, and $e=18 n-11$. Now, we define the labeling $\lambda: V(G) \rightarrow\{1,2, \ldots, v\}$ as follows:

$$
\lambda(c)=10 n-2 .
$$

For $\quad 1 \leq l_{i} \leq n_{i}$ odd;

$$
\lambda(u)= \begin{cases}\frac{l_{1}+1}{2}, & \text { for } u=x_{1}^{l_{1}}, \\ (n+2)-\frac{l_{2}+1}{2}, & \text { for } u=x_{2}^{l_{2}}, \\ (n+1)+\frac{l_{3}+1}{2}, & \text { for } u=x_{3}^{l_{3}}, \\ (2 n+3)-\frac{l_{4}+1}{2}, & \text { for } u=x_{4}^{l_{4}} \\ (3 n+3)-\frac{l_{5}+1}{2}, & \text { for } u=x_{5}^{l_{5}}, \\ (5 n+2)-\frac{l_{6}+1}{2}, & \text { for } u=x_{6}^{l_{6}} \\ (9 n-1)-\frac{l_{7}+1}{2}, & \text { for } u=x_{7}^{l_{7}} .\end{cases}
$$

For $\quad 2 \leq l_{i} \leq n_{i}-1$ even;

$$
\lambda(u)= \begin{cases}(9 n-2)+\frac{l_{1}}{2}, & \text { for } u=x_{1}^{l_{1}}, \\ (10 n-2)-\frac{l_{2}}{2}, & \text { for } u=x_{2}^{l_{2}}, \\ (10 n-2)+\frac{l_{3}}{2}, & \text { for } u=x_{3}^{l_{3}}, \\ (11 n-2)-\frac{l_{4}}{2}, & \text { for } u=x_{4}^{l_{4}}, \\ (12 n-3)-\frac{l_{5}}{2}, & \text { for } u=x_{5}^{l_{5}}, \\ (14 n-5)-\frac{l_{6}}{2}, & \text { for } u=x_{6}^{l_{6}} \\ (18 n-9)-\frac{l_{7}}{2}, & \text { for } u=x_{7}^{l_{7}} .\end{cases}
$$

The set of all edge-sums generated by the above formulas forms a consecutive integer sequence $s=9 n, 9 n+1, \cdots, 9 n-1+e$. Therefore, by Proposition $1.1, \lambda$ can be extended to a super $(a, 0)$-EAT labeling with magic constant $a=2 v+s-1=45 n-21$ and to a super $(a, 2)$-EAT labeling with minimum edge-weight $a=v+1+s=$ $27 n-9$.

Theorem 2.5. For any odd $n \geq 3, G \cong T(n, n, n, n, 2 n-1,4 n-3,8 n-7)$ admits a super $(a, 1)$-EAT labeling with $a=s+\frac{3 v}{2}$, where $v=|V(G)|$ and $s=9 n$.

Proof. Let us consider the vertex and edge set of $G$ and the labeling $\lambda: V(G) \rightarrow$ $\{1,2, \ldots, v\}$ by the same manner as in Theorem 2.4. It follows that edge-sums of all the edges of $G$ constitute an arithmetic sequence $9 n, 9 n+1, \cdots, 9 n-1+e$, with 
common difference 1 . We denote it by $A=\left\{a_{i} ; 1 \leq i \leq e\right\}$. Now to show that $\lambda$ is an $(a, 1)$-EAT labeling of $G$, define the set of edge-labels as $B=\left\{b_{j}=v+j ; 1 \leq\right.$ $j \leq e\}$. The set of edge-weights can be obtained as $C=\left\{a_{2 i-1}+b_{e-i+1} ; 1 \leq i \leq\right.$ $\left.\frac{e+1}{2}\right\} \cup\left\{a_{2 j}+b_{\frac{e-1}{2}-j+1} ; 1 \leq j \leq \frac{e+1}{2}-1\right\}$. It is easy to see that $C$ constitutes an arithmetic sequence with $d=1$ and $a=s+\frac{3 v}{2}=36 n-15$. Since all vertices receive the smallest labels, $\lambda$ is a super $(a, 1)$-EAT labeling.

Theorem 2.6. For any $n \geq 3$ odd, $G \cong T\left(n, n, n, n, n_{5}, \ldots, n_{r}\right)$ admits a super $(a, 0)$-EAT labeling with $a=2 v+s-1$ and a super $(\dot{a}, 2)$-EAT labeling with $\dot{a}=v+s+1$ where $v=|V(G)|, s=(2 n+4)+\sum_{m=5}^{r}\left[2^{m-5}(n-1)+1\right], r \geq 5$ and $n_{m}=2^{m-4}(n-1)+1$ for $5 \leq m \leq r$.

Proof. Let us denote the vertices and edges of $G$, as follows:

$V(G)=\{c\} \cup\left\{x_{i}^{l_{i}} \mid 1 \leq i \leq r ; 1 \leq l_{i} \leq n_{i}\right\}, E(G)=\left\{c x_{i}^{1} \mid 1 \leq i \leq r\right\} \cup$ $\left\{x_{i}^{l_{i}} x_{i}^{l_{i}+1} \mid 1 \leq i \leq r ; 1 \leq l_{i} \leq n_{i}-1\right\}$. If $v=|V(G)|$ and $e=|E(G)|$ then $v=$ $(4 n+1)+\sum_{m=5}^{r}\left[2^{m-4}(n-1)+1\right]$ and $e=v-1$. Now, we define the labeling $\lambda: V(G) \rightarrow\{1,2, \ldots, v\}$ as follows:

$$
\lambda(c)=(3 n+2)+\sum_{m=5}^{r}\left[2^{m-5}(n-1)+1\right] .
$$

For $\quad 1 \leq l_{i} \leq n_{i}$ odd, where $i=1,2,3,4$ and $5 \leq i \leq r$, we define

$$
\begin{gathered}
\lambda(u)= \begin{cases}\frac{l_{1}+1}{2}, & \text { for } u=x_{1}^{l_{1}}, \\
(n+2)-\frac{l_{2}+1}{2}, & \text { for } u=x_{2}^{l_{2}}, \\
(n+1)+\frac{l_{3}+1}{2}, & \text { for } u=x_{3}^{l_{3}}, \\
(2 n+3)-\frac{l_{4}+1}{2}, & \text { for } u=x_{4}^{l_{4}} .\end{cases} \\
\lambda\left(x_{i}^{l_{i}}\right)=(2 n+3)+\sum_{m=5}^{i}\left[2^{m-5}(n-1)+1\right]-\frac{l_{i}+1}{2} \text { respectively. }
\end{gathered}
$$

Let $\alpha=(2 n+2)+\sum_{m=5}^{r}\left[2^{m-5}(n-1)+1\right]$. For $\quad 2 \leq l_{i} \leq n_{i}$ even, and $1 \leq i \leq r$, we define 


$$
\lambda(u)= \begin{cases}\alpha+\frac{l_{1}}{2}, & \text { for } u=x_{1}^{l_{1}}, \\ (\alpha+n)-\frac{l_{2}}{2}, & \text { for } u=x_{2}^{l_{2}}, \\ (\alpha+n)+\frac{l_{3}}{2}, & \text { for } u=x_{3}^{l_{3}}, \\ (\alpha+2 n)-\frac{l_{4}}{2}, & \text { for } u=x_{4}^{l_{4}} .\end{cases}
$$

and

$$
\lambda\left(x_{i}^{l_{i}}\right)=(\alpha+2 n)+\sum_{m=5}^{i}\left[2^{m-5}(n-1)\right]-\frac{l_{i}}{2} .
$$

The set of all edge-sums generated by the above formulas forms a consecutive integer sequence $s=\alpha+2, \alpha+3, \cdots, \alpha+1+e$. Therefore, by Proposition 1.1, $\lambda$ can be extended to a super $(a, 0)$-EAT labeling with magic constant $a=v+e+s=$ $2 v+(2 n+3)+\sum_{m=5}^{r}\left[2^{m-5}(n-1)+1\right]$ and to a super (á, 2)-EAT labeling with minimum edge-weight $a ́ a+1+s=v+(2 n+5)+\sum_{m=5}^{r}\left[2^{m-5}(n-1)+1\right]$.

Theorem 2.7. For any $n \geq 3$ odd, $G \cong T\left(n, n, n, n, n_{5}, \ldots, n_{r}\right)$ admits super $(a, 1)$ EAT labeling with $a=s+\frac{3 v}{2}$ if $v$ is even, where $v=|V(G)|, s=(2 n+4)+$ $\sum_{m=5}^{r}\left[2^{m-5}(n-1)+1\right], r \geq 5$, and $n_{m}=2^{m-4}(n-1)+1$ for $5 \leq m \leq r$.

Proof. Let us consider the vertex and edge set of $G$ and the labeling $\lambda: V(G) \rightarrow$ $\{1,2, \ldots, v\}$ by the same manner as in Theorem 2.6. It follows that edge-sums of all the edges of $G$ constitute an arithmetic sequence $s=\alpha+2, \alpha+3, \cdots, \alpha+1+e$ with common difference 1 , where $\alpha=(2 n+2)+\sum_{m=5}^{r}\left[2^{m-5}(n-1)+1\right]$. We denote it by $A=\left\{a_{i} ; 1 \leq i \leq e\right\}$. Now to show that $\lambda$ is an $(a, 1)$-EAT labeling of $G$, define the set of edge-labels as $B=\left\{b_{j}=v+j ; 1 \leq j \leq e\right\}$. The set of edge-weights can be obtained as $C=\left\{a_{2 i-1}+b_{e-i+1} ; 1 \leq i \leq \frac{e+1}{2}\right\} \cup\left\{a_{2 j}+b_{\frac{e-1}{2}-j+1} ; 1 \leq j \leq \frac{e+1}{2}-1\right\}$. It is easy to see that $C$ constitutes an arithmetic sequence with $d=1$ and $a=s+\frac{3 v}{2}$. Since, all vertices receive the smallest labels, $\lambda$ is a super $(a, 1)$-EAT labeling.

From Theorems 2.1, 2.3, 2.4 and 2.6 by the principal of duality it follows that we can find the super $(a, 0)$-EAT labelings with different magic constant. Thus, we have the following corollaries:

Corollary 2.1. For any odd $n \geq 3, T(n, n, n, n, 2 n-1)$ admits a super $(a, 0)$-EAT total labeling with magic constant $a=15 n-1$. 
Corollary 2.2. For any odd $n \geq 3, T(n, n, n, n, 2 n-1,4 n-3)$ admits a super $(a, 0)$-EAT labeling with magic constant $a=25 n-9$.

Corollary 2.3. For any odd $n \geq 3, T(n, n, n, n, 2 n-1,4 n-3,8 n-7)$ admits a super $(a, 0)$-EAT labeling with magic constant $a=45 n-27$.

Corollary 2.4. For any $n \geq 3$ odd, and $r \geq 5, T\left(n, n, n, n, n_{5}, \ldots, n_{r}\right)$ admits a super $(a, 0)$-EAT total labeling with $a=3 v-(2 n+1)-\sum_{m=5}^{r}\left[2^{m-5}(n-1)+1\right]$, where $n_{m}=2^{m-4}(n-1)+1$ for $5 \leq m \leq r$.

\section{CONCLUSION}

In this paper, we have proved that a subclass of subdivided stars denoted by $T\left(n, n, n, n, n_{5}, \ldots, n_{r}\right)$, admits super $(a, d)$-EAT labelings for $d=0,1,2$, when $n \geq 3$ is odd, $r \geq 5$ and $n_{m}=2^{m-4}(n-1)+1$ for $5 \leq m \leq r$.

Acknowledgement. The research contents of this paper are partially supported by the Higher Education Commission (HEC) of Pakistan and National University of Computer and Emerging Sciences (NUCES) Lahore, Pakistan.

\section{References}

[1] Bača, M., Lin, Y., and Muntaner-Batle, F.A., "Edge-antimagic labeling of forests", Utilitas Math., 81 (2010), 31-40.

[2] Bača, M., Lin, Y., Miller, M., and Youssef, M.Z., "Edge-antimagic graphs", Discrete Math., 307 (2007), 1232-1244.

[3] Bača, M., Lin, Y., Miller, M., and Simanjuntak, R., "New constructions of magic and antimagic graph labelings", Utilitas Math., 60 (2001), 229-239.

[4] Bača, M., Lin, Y., and Muntaner-Batle, F.A., "Super edge-antimagic labelings of the pathlike trees", Utilitas Math., 73 (2007), 117-128.

[5] Bača, M., and Miller, M., Super Edge-Antimagic Graphs, Brown Walker Press, Boca Raton, Florida USA, 2008.

[6] Bača, M., Semaničová -Feňovčíková, A., and Shafiq, M.K., "A method to generate large classes of edge-antimagic trees", Utilitas Math., 86 (2011), 33-43.

[7] Baskoro, E.T., and Ngurah, A.A.G., "On super edge-magic total labelings", Bull. Inst. Combin. Appil., 37 (2003), 82-87.

[8] Baskoro, E.T., Sudarsana, I.W., and Cholily, Y.M., "How to construct new super edge-magic graphs from some old ones", J. Indones. Math. Soc. (MIHMI), 11:2 (2005), 155-162.

[9] Enomoto, H., Llado, A.S., Nakamigawa, T., and Ringel, G., "Super edge-magic graphs", SUT J. Math., 34 (1980), 105-109.

[10] Figueroa-Centeno, R.M., Ichishima, R., and Muntaner-Batle, F.A., "The place of super edgemagic labeling among other classes of labeling", Discrete Math., 231 (2001), 153-168.

[11] Figueroa-Centeno, R.M., Ichishima, R., and Muntaner-Batle, F.A., "On super edge-magic graph", Ars Combin., 64 (2002), 81-95.

[12] Fukuchi, Y., "A recursive theorem for super edge-magic labeling of trees", SUT J. Math., 36 (2000), 279-285.

[13] Gallian, J.A., "A dynamic survey of graph labeling", Electron. J. Combin., (2012).

[14] Hussain, M., Baskoro, E.T., and Slamin, "On super edge-magic total labeling of banana trees", Utilitas Math., 79 (2009), 243-251. 
[15] Javaid, M., Bhatti, A.A., Hussain, M., "On $(a, d)$-edge-antimagic total labeling of extended w-trees", Utilitas Math., 87 (2012), 293-303.

[16] Javaid, M., Hussain, M., Ali, K., and Shaker, H., "Super edge-magic total labeling on subdivision of trees", Utilitas Math., 89 (2012), 169 - 177.

[17] Javaid, M., and Bhatti, A.A., On super $(a, d)$-edge-antimagic total labeling of subdivided stars. Ars Combin., 105 (2012), 503-512.

[18] Javaid, M., Hussain, M., Ali, K., and Dar, K.H., "Super edge-magic total labeling on w-trees", Utilitas Math., 86 (2011), 183-191.

[19] Javaid, M., Labeling Graphs and Hypergraphs, PhD Thesis FAST-NUCES, Lahore Campus, Pakistan, 2013

[20] Kotzig, A., and Rosa, A., Magic valuations of finite graphs, Canad. Math. Bull., 13 (1970), 451-461.

[21] Kotzig, A., and Rosa, A., Magic valuation of complete graphs, Centre de Recherches Mathematiques, Universite de Montreal, 1972, CRM-175.

[22] Lee, S.M., and Shah, Q.X., "All trees with at most 17 vertices are super edge-magic", 16th MCCCC Conference, Carbondale, University Southern Illinois, (2002).

[23] Lu, Y.J., "A proof of three-path trees $P(m, n, t)$ being edge-magic", College Mathematica, $\mathbf{1 7 : 2}(2001), 41-44$.

[24] Lu, Y.J., " A proof of three-path trees $P(m, n, t)$ being edge-magic (II)", College Mathematica, 20:3 (2004), 51-53.

[25] Ngurah, A.A.G., Simanjuntak, R., and Baskoro, E.T., "On (super) edge-magic total labeling of subdivision of $K_{1,3}$ ", SUT J. Math., 43 (2007), 127-136.

[26] Salman, A.N.M., Ngurah, A.A.G., and Izzati, N., On "Super Edge-Magic Total Labeling of a Subdivision of a Star $S_{n}$ ", Utilitas Mthematica, 81 (2010), 275-284.

[27] Simanjuntak, R., Bertault, F., and Miller, M., "Two new $(a, d)$-antimagic graph labelings", Proc. of Eleventh Australasian Workshop on Combinatorial Algorithms, (2000), 179-189.

[28] Sugeng, K.A., Miller, M., Slamin, and Bača, M., " $(a, d)$-edge-antimagic total labelings of caterpillars", Lecture Notes Comput. Sci., 3330 (2005), 169-180.

[29] West, D.B., An Introduction to Graph Theory Prentice-Hall, 1996.

[30] Wallis, W.D., Magic Graphs, Birkhäuser, Boston-Basel-Berlin, 2001. 